Cys-LTs, derived from arachidonic acid, are produced in excess in patients with aspirin-induced asthma (AIA). In these patients, the key enzyme, LTC $_{4}$ synthase, is overexpressed in bronchi, and its mRNA is upregulated in peripheral blood eosinophils. ${ }^{1}$ Cys-LTs mediate their biological actions through at least 2 distinct receptors $^{2}$; these are designated $c y s-L T_{1}$ and $c y s-L T_{2}$.

Montelukast, a drug recently introduced for the treatment of asthma, ${ }^{3}$ is a specific antagonist of cys-LT ${ }_{1}$. An antagonist of cys-LT 2 is not available. In isolated rabbit heart, myocardial ischemia develops as a result of enhanced cys-LT formation, originating from the transfer of leukotriene $\mathrm{A}_{4}$ from granulocytes to endothelial cells. ${ }^{4}$ Atherosclerosis is associated with the appearance of a leukotriene receptor capable of inducing hyperreactivity of human epicardial coronary arteries in response to cys-LTs. ${ }^{5}$ Increased cys-LT levels have been described in the urine of patients with coronary artery disease ${ }^{6}$ and in coronary blood of patients undergoing angioplasty. ${ }^{7}$

We investigated a 37 -year-old man without coronary risk factors who 3 years earlier had developed AIA. The condition was controlled by chronic inhaled corticosteroids and oral prednisone $6 \mathrm{mg}$. Oral aspirin challenge results were positive: aspirin at a cumulative dose of $118 \mathrm{mg}$ precipitated dyspnea with a fall in $\mathrm{FEV}_{1}$ of $35 \%$, nasal discharge, headache, and nausea; 2 to 4 hours after the reaction there was an 8 -fold $(800 \%)$ increase in urinary leukotriene $\mathrm{E}_{4}$ $\left(\mathrm{LTE}_{4}\right)$ and a $70 \%$ increase in the stable prostaglandin $\mathrm{D}_{2}$ metabolite $9 \alpha 11 \beta$ PGF2. In contrast, rofecoxib $50 \mathrm{mg}$, a specific Cox-2 inhibitor, was very well tolerated. At base, the $\mathrm{LTE}_{4}$ urinary excretion, measured on several occasions, was very high (1500-5000 $\mathrm{pg} / \mathrm{mg}$ creatinine), ranking the patient in the upper $10 \%$ of 180 subjects with AIA who were studied by us.

A year before entry, the patient had begun to experience episodes of retrosternal chest pain. With a frequency of 1 to 4 times per week, these occurred when the patient was at rest, each episode lasting approximately 1 minute. The only electrocardiographic abnormality was flat $\mathrm{T}$ waves in aVL. Holter monitoring for 24 hours showed short incidents of supraventricular tachycardia $(<150 / \mathrm{min})$ and 5 episodes of myocardial ischemia with ST depression and T wave inversion, each lasting 15 to 45 seconds. A coronary artery angiogram showed normal coronary arteries, and cardiac echo was normal.

Because the patient produced excessive amounts of cys-LTs, we investigated the possible role of cys- $\mathrm{LT}_{1}$ in his myocardial ischemia. We performed 4 exercise tests with the patient on a treadmill (modified Bruce protocol). The patient was premedicated with either montelukast $10 \mathrm{mg}$ or placebo 90 minutes before each exercise test and was blinded to the nature of the therapy.

Results of the 2 placebo tests were negative; the patient completed the protocol with no electrocardiographic ischemic changes. However, when montelukast was given to the patient before the exercise testing, the protocol had to be terminated sooner because of chest pain and changes indicating myocardial ischemia: there was ST-segment depression in leads I, aVL, and $\mathrm{V}_{3-6}$, and there were recurrent episodes of venticular tachycardia, necessitating treatment in the intensive care unit.

Blood was drawn before the exercise stress test and then every 15 minutes for 2 hours after the test for measurement of tryptase and $9 \alpha 11 \beta \mathrm{PGF}_{2}$ by gas chromatography/mass spectrometry; urine was collected over a period of 4 hours for $\mathrm{LTE}_{4}$ (ELISA).

Results of the placebo-controlled tests showed a rise only in $9 \alpha 11 \mathrm{PGFF}_{2}$; this rise, from 7.2 to $12.5 \mathrm{ng} / \mathrm{mL}$, occurred by $45 \mathrm{~min}$ utes. The exercise tests preceded by montelukast were accompanied by a rise in $9 \alpha 11 \beta \mathrm{PGF}_{2}$ from 6.4 to $14.5 \mathrm{ng} / \mathrm{mL}$ and a rise in tryptase from 6.0 to $7.3 \mathrm{~g} / \mathrm{L}$ and $\mathrm{LTE}_{4}$ (Table I), indicating discharge of cys-LTs and enhanced mast cell activation.

Given that the patient's myocardial ischemia was mediated by cys-LTs, he was treated for 4 weeks with a 5-lipoxygenase inhibitor (Zileutone) at a daily dose of $4 \times 600 \mathrm{mg}$. This therapy led to an

\section{Myocardial ischemia possibly mediated by cysteinyl leukotrienes}

To the Editor:

Ischemic heart disease manifests itself clinically as a series of syndromes, the pathophysiologic causes of which must be understood if specific treatments are to be designed for specific causes of decreased myocardial oxygenation. We here report that cysteinyl leukotrienes (cys-LTs) can be involved as a cause of myocardial ischemia. 
TABLE I. Urinary $\mathrm{LTE}_{4}$ excretion (ng/mg creatinine) after exercise stress testing

\begin{tabular}{lccc}
\hline & \multicolumn{2}{c}{ No Zileutone } & \multicolumn{1}{c}{ Following Zileutone } \\
\cline { 2 - 4 } Time of urine collection & Pretreatment with placebo & Pretreatment with montelukast & Pretreatment with montelukast \\
\hline At base & 1804 & 1523 & 529 \\
$0-2 \mathrm{~h}$ & 2098 & 1880 & 143 \\
$2-4 \mathrm{~h}$ & 1574 & 2652 & 143 \\
\hline
\end{tabular}

Placebo or 10 mg montelukast was given 90 minutes before each test.

improvement in $\mathrm{FEV}_{1}$ of $12 \%$, clearing of the nose, return of the sense of smell, disappearance of the retrosternal pain, and a fall in urinary $\mathrm{LTE}_{4}$ from 3000 to $450 \mathrm{ng} / \mathrm{mg}$ creatinine. Holter monitoring revealed no ischemic episodes. Results of repeated exercise stress testing preceded by $10 \mathrm{mg}$ montelukast showed no evidence of ischemia; blood and urinary parameters studied showed no changes.

This patient had AIA, a distinct clinical syndrome that affects 5\% to $10 \%$ of adults with asthma but remains largely underdiagnosed. ${ }^{1}$ At the biochemical level, AIA is characterized by a chronic overproduction of cys-LTs; our patient excreted very large quantities of $\mathrm{LTE}_{4}$ in urine, a parameter considered to reflect global cys-LT production. For the following reasons, we believe that massive cys-LT overproduction was the cause of his myocardial ischemia: (1) the classical coronary risk factors were absent; (2) coronary arteries were free of obstruction or other signs of atherosclerosis; (3) attacks of ischemia were accompanied by further increases in urinary $\mathrm{LTE}_{4}$, already elevated at base; (4) treatment with a 5-LO inhibitor led to a dramatic fall in cys-LT production and disappearance of myocardial ischemia.

We used montelukast as a probe with which to understand the role of the cys- $\mathrm{LT}_{1}$ receptor in this case. Unexpectedly, the drug precipitated myocardial ischemia during the exercise stress test. Perhaps montelukast, by blocking cys-LT ${ }_{1}$, made more cys-LTs available for binding at the second receptor, cys- $\mathrm{LT}_{2}$, which in contrast with cys- $\mathrm{LT}_{1}$ is expressed in the heart.2,6

Cys-LTs possess a unique pharmacologic profile characterized by potent constriction of microvasculature; they can enhance permeability, reduce coronary blood flow, and reduce myocardial contractility and cardiac output without affecting the heart rate.$^{8}$ It has been hypothesized ${ }^{3,6}$ that these actions, per se, could one day explain the pathologic process in some cases of angina. Indeed, our report indicates that myocardial ischemia might be caused by enhanced biosynthesis of cys-LTs. Pharmacologic inhibition of cysLT production— but not blockade of the cys-LT 1 receptor-leads to regression of ischemia.

Andrew Szczeklik, MD, PhD Ewa Niżankowska, $M D, P h D$ Lucyna Mastalerz, MD, PhD Grażyna Bochenek, $M D, P h D$ Jagellonian University School of Medicine Department of Medicine ul. Skawińska 8 31-066 Kraków, Poland

We thank Professor John Martin for inspiring discussion.

\section{REFERENCES}

1. Szczeklik A, Stevenson DD. Aspirin-induced asthma: advances in pathogenesis and management. J Allergy Clin Immunol 1999;104:5-13.

2. Heise CE, O’Dowl BF, Figueroa DJ, Sawyer N, Nguyen T, Im DS, et al. Characterization of the human cysteinyl leukotriene 2 receptor. J Biol Chem 2000;275:30531-6.

3. Folco G. Inhibition of leukotrienes: an overviev. In: Folco G, Samuelsson
B, Murphy RC, editors. Novel inhibitors of leukotrienes. Basel: Birkhauser Verlag; 1999. p. 205-14.

4. Sala A, Aliev GM, Rossini G, Berti F, Buccelati C, Burnstock G, et al. Morphological and functional changes of coronary vasculature caused by transcellular biosynthesis of sulfodipeptide leukotrienes in isolated heart of rabbit. Blood 1996;87:1824-32.

5. Allen S, Dashwood M, Morrison K, Yacoub M. Different leukotriene constrictor responses in human atherosclerotic coronary arteries. Circulation 1998;97:2406-13.

6. Sampson AP. Leukotrienes in cardiovascular disease. Clin Exp Allergy Rev 2001;1:170-4.

7. Brezinski DA, Nesto RW, Serhan CN. Angioplasty triggers intracoronary leukotrienes and lipoxin A4. Impact of aspirin therapy. Circulation 1992;86:56-63.

8. Vigorito C, Giordano A, Cirillo R, Genovese A, Rengo F, Marone G. Metabolic and hemodynamic effects of peptido leukotriene C4 and D4 in man. Int J Clin Lab Res 1997;27:178-84. 